MATEC Web of Conferences 22,05012 (2015)

DOI: $10.1051 /$ matecconf/ 20152205012

(C) Owned by the authors, published by EDP Sciences, 2015

\title{
The Difference Format of Landau-Lifshitz Equation in Two-dimensional Case
}

Taiyong Zhong

Department of Maths and Finance, Yunyang Teachers' College, Shiyan, Hubei, China

ABSTRACT: In this paper, the author considers a difference scheme of Laudau-Lifshitz equation (LL for short and modulus of $u_{j}^{n}$ which are constantly remaining equal to 1 . Using this iteration format error which is ordered to $\frac{t}{2 h^{2}}$, the author comes to a conclusion based on several initial simulations. According to some conditions, the author gives the numerical solution, the examples of exact solution and the error comparisons of the solutions.

Keywords: two-dimensional Landau-Lifshitz Equation; numerical Convergence; blow up; numerical examples

\section{INTRODUCTION}

The continuous Heisenberg spin chain has aroused considerable interest among physicists in the classical study of one-dimensional motion of ferromagnetic chain. In 1907, Weiss explained the property of the ferromagnetic bodies through a study about elements of the hypothesis. In 1928, Heisenberg explained the molecular field theory which is proffered by Weiss. In 1935, L. D. Landau and E. M. Lifshitz proposed the following equation [1]:

$\frac{\partial u}{\partial t}=\lambda_{1} u \times H^{e}-\lambda_{2} u \times\left(u \times H^{e}\right)$

Where the effect field $H^{e}$ was defined as follows:

$H^{e} \equiv-\frac{\partial}{\partial u} E_{m g}(u)=H-\nabla(\varphi(u))+\sum_{i, j=1}^{3} a_{i j} \frac{\partial^{2} u}{\partial x_{i} \partial x_{j}}$

When the external magnetic field is vanish, the L-L equation is shown as follows:

$\frac{\partial u}{\partial t}=\lambda_{1} u \times \Delta u-\lambda_{2} u \times(u \times \Delta u)$

Where $\lambda_{1}, \lambda_{2}$ are constants, $\lambda_{2}>0$. The equation describes an evolution of spin fields in continuum ferromagnetic body in a domain $\Omega \subset R^{n}$. It performs a fundamental role in the research of non-equilibrium magnetism.

A lot of work on the Landau-Lifshitz system of 1-dimensional motion spin chain has been made by some physicists and mathematicians.

The energy critical Schrodinger map is related to the Landau-Lifshitz equation for ferromagnetism, and it belongs to a class of geometric evolution equations including wave maps and the harmonic heat flow. It has attracted a considerable attention in the past ten years.

$$
\left\{\begin{array}{l}
u_{t}=u \times \Delta u, u(x, t) \in S^{2},(x, t) \in R^{2} \times R^{+}, \\
\left.u\right|_{t=0}=u_{0} \in H^{1}
\end{array}\right.
$$

So will the solutions of minimization problem on the energy function of Landau-Lifshitz equation be smooth? In 2000, Nai-Heng Chang, J. Shatah and K. Uhlenbeck considered an initial value problem for the two-dimensional cylindrical symmetric Landau-Lifshitz equations [2]. There are some papers to consider the partial regularity and the existence of weak solutions [3], [4], analyze the concentration sets [5] study the relation between weak solution and harmonic map [5] [6] and discuss the stability.

Whether there exists the global smooth solution for Landau-Lifshitz equation is an open problem. Indeed, the multidimensional Landau-Lifshitz equation is hard to solve exactly, even two-dimensional Heisenberg's equations. Only when there is a lack of the external field or the anisotropy field, many authors had attempted Hirota's famous method, Lie algebraic structure, and Backlund transform, Double transform and so on, but exact solutions were still not established.

In 2000-2001, Guo, Han and Yang established several exact solutions of the cylindrical symmetric n-dimension Landau-Lifshitz equation in [7] and [8] In 2001, Guo and Yang constructed a family of exact disc solutions of the two-dimensional Landau-Lifshitz equation in [9].

In 2003, Yang and Chang obtained an exact transform between with an external field and without an external field [10]. Based on the transform, some sufficient and necessary conditions from the solution with an external field converges to a certain solution without an external field were given [10].

When both external field and anisotropy field are presented, there is no other paper discussed the explicit solution of the multi-dimensional Landau-Lifshitz equation until 2008. In 2009, we researched the mul- 
ti-dimensional Landau-Lifshitz equation with an uprush external field and anisotropy field. We gave a method to find explicit solutions and provided some explicit solutions for two-dimensional case [11]. $\mathrm{Mu}$ and Liu et. gave a method to find that an external field and anisotropy field such as the solution remained unvarying [12].

In 2011, we presented a method to solve the Landau-Lifshitz equations with the external magnetic field and anisotropy field. Using the method, we obtained a new family of symmetric solutions, that is, the spherical cone symmetric solution. We also showed how those solutions can evolve with time and gave a series of explicit dynamic spherical cone symmetric solutions [13].

\section{DIFFERENCE FORMAT OF LAN- DAU-LIFSHITZ EQUATION}

First, we only consider the initial-boundary condition of (4) in rectangular domain $Q_{T}=0 \leq x \leq l ; 0 \leq t \leq T$ :

$\left\{\begin{array}{l}u(0, t)=u(l, t)=0, t \in[0, T] \\ u(x, 0)=\varphi(x), x \in[0, l]\end{array}\right.$

Second, we have given numerical examples and the error comparisons of the solutions in the Landau-Lifshitz equation.

The continuous Heisenberg spin chain has aroused considerable interest among physicists. In the classical study of one-dimensional motion of ferromagnetic chain, the so-called Landau-Lifshitz equation [1] for the isotropic Heisenberg chain is a special case in the following system:

$z_{t}=z \times z_{x x}+f(x, t, z)$

And the following system with the Gilbert damping term is shown as follows:

$z_{t}=-\alpha z \times\left(z \times z_{x x}\right)+z \times z_{x x}+f(x, t, z)(7)$

Where " $x$ " denotes the vector cross product in $R^{3}, \quad \alpha>0$ is the Gilbert damping constant, $z=\left(z_{1}, z_{2}, z_{3}\right): R \times R^{+} \rightarrow R^{3}$ is the unknown vector-valued function. There have been many contributions to study the initial value problem with periodic boundary condition for a class of general systems in the ferromagnetic chain [14]

$$
z_{t}=-\alpha z \times\left(z \times z_{x x}\right)+z \times z_{x x}+z \times f(z)
$$

In this paper, we want to establish sooth solutions of the initial value problems for one class of general systems of the ferromagnetic chain, which takes the form as follows: $z_{t}=z \times z_{x x}+z \times A z$

Where, $A=\operatorname{diag}\{1,2,3\}$. It is with the periodic boundary condition and the initial condition as follows:

$z(x-D, t)=z(x+D, t), \quad D>0$

$z(x, 0)=z^{0}(x)$

Here the given function $z^{0}(x)$ satisfies as follows:

$z^{0}(x-D)=z^{0}(x+D),\left|z^{0}(x)\right|=1, \forall x \in R$.

We also study the initial value problem with the Gilbert damping term as follows:

$z_{t}=-\alpha z \times\left(z \times z_{x x}\right)+z \times z_{x x}+z \times A z$

Then we obtain it as follows:

$\left\{\begin{array}{l}\frac{\partial u}{\partial t}=\alpha \frac{\partial^{2} u}{\partial x^{2}}+v \frac{\partial^{2} w}{\partial x^{2}}-w \frac{\partial^{2} v}{\partial x^{2}}+\alpha u\left(\frac{\partial u}{\partial x}\right)^{2}+w v \\ \frac{\partial v}{\partial t}=\alpha \frac{\partial^{2} v}{\partial x^{2}}+w \frac{\partial^{2} u}{\partial x^{2}}-u \frac{\partial^{2} w}{\partial x^{2}}+\alpha v\left(\frac{\partial v}{\partial x}\right)^{2}-2 w u \\ \frac{\partial w}{\partial t}=\alpha \frac{\partial^{2} w}{\partial x^{2}}+u \frac{\partial^{2} v}{\partial x^{2}}-v \frac{\partial^{2} u}{\partial x^{2}}+\alpha w\left(\frac{\partial w}{\partial x}\right)^{2}+u v\end{array}\right.$

Set $\alpha=1$, let $\frac{\tau}{h^{2}}=\lambda$, where $\tau$ is time steps and

$h$ is space-step size.

Then (13) changes as follows:

$u\left(x_{j}, t_{k+1}\right)-u\left(x_{j}, t_{k}\right)=\lambda\left(u\left(x_{j+1}, t_{k}\right)-2 u\left(x_{j}, t_{k}\right)+u\left(x_{j-1}, t_{k}\right)\right.$

$+v\left(x_{j}, t_{k}\right)\left(w\left(x_{j+1}, t_{k}\right)+w\left(x_{j-1}, t_{k}\right)\right)-w\left(x_{j}, t_{k}\right)\left(v\left(x_{j+1}, t_{k}\right)\right.$

$\left.\left.\left.+v\left(x_{j-1}, t_{k}\right)\right)+u\left(x_{j}, t_{k}\right)\left(u\left(x_{j+1}, t_{k}\right)-u\left(x_{j}, t_{k}\right)\right)^{2}\right)+w\left(x_{j}, t_{k}\right) v\left(x_{j}, t_{k}\right)\right)$

$z=(u, v, w)=\left(\sqrt{1-s_{0}^{2}} \cos \xi, \sqrt{1-s_{0}^{2}} \sin \xi, s_{0}\right)$

Where $\xi=k x-w t+\varphi, s_{0}, h, w, \varphi$ are constants.

\section{NUMERICAL TESTS}

In order to verify the theoretical results, we come to a conclusion by several initial simulations.

3.1 First, for the initial-value problem, this is shown as follows:

$\left\{\begin{array}{l}u_{t}=u \times \Delta u,(t, x) \in R^{2} \times R_{+}, \\ \left.u\right|_{t=0}=u_{0}, \\ u(t, x) \in S^{2} .\end{array}\right.$ 
ICETA 2015

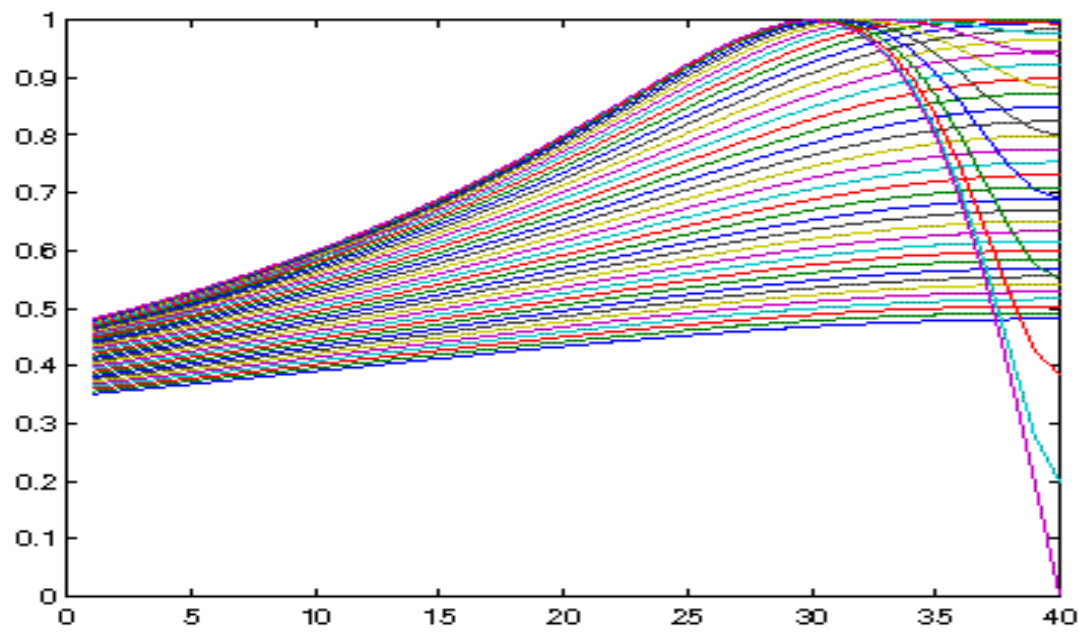

(a) Initial-value condition $u_{1}$

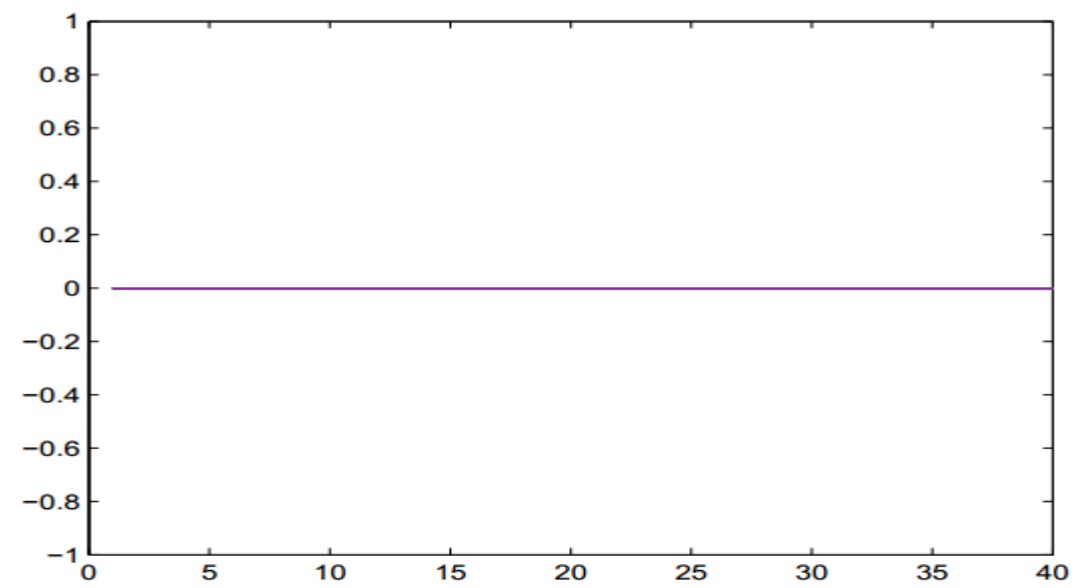

(b) Initial-value condition $u_{2}$

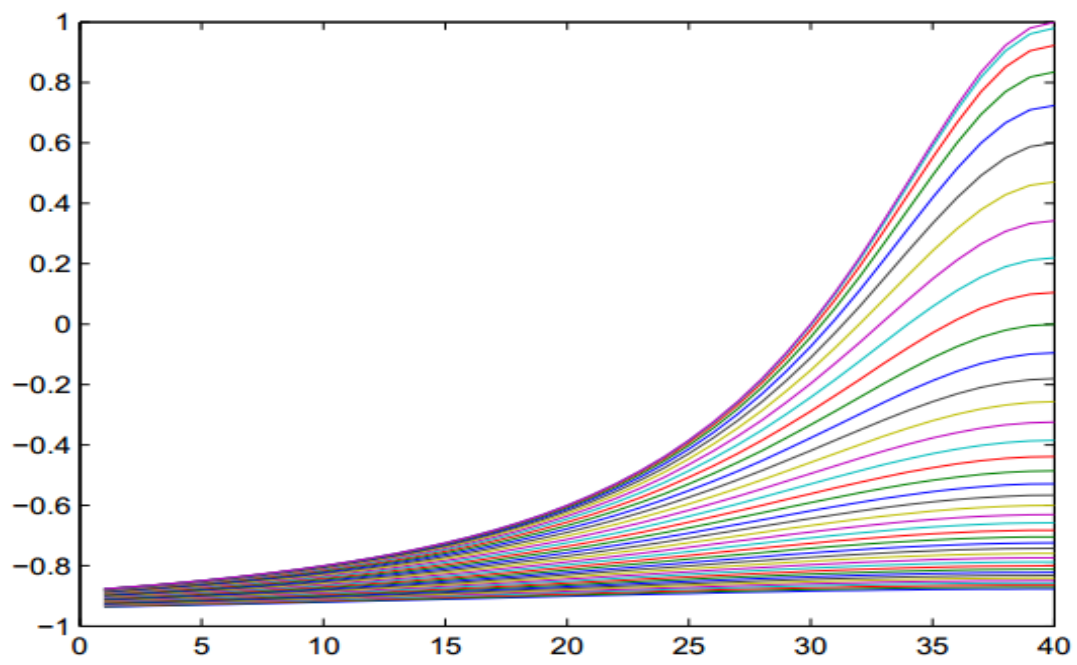

(c) Initial-value condition $u_{3}$

Figure 1.the irregular change of initial value 

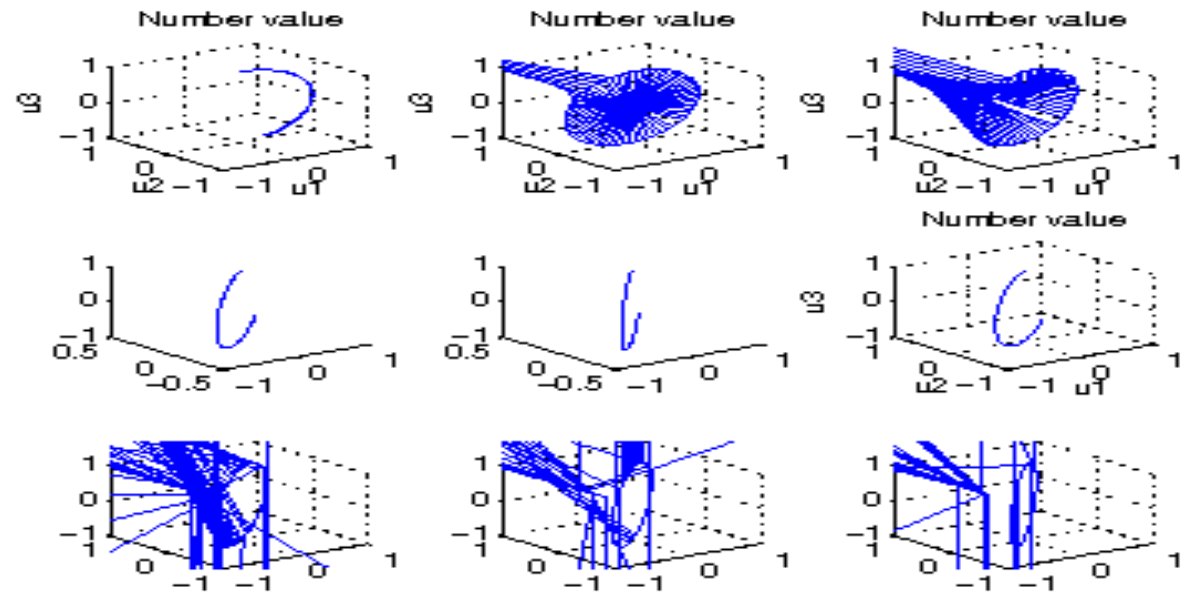

Figure 2. Number value

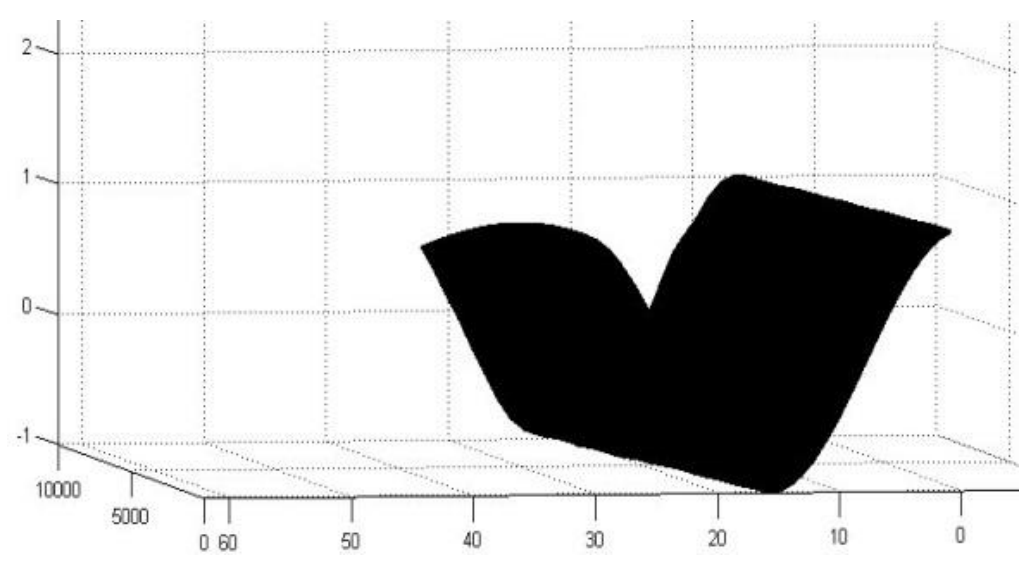

(a)

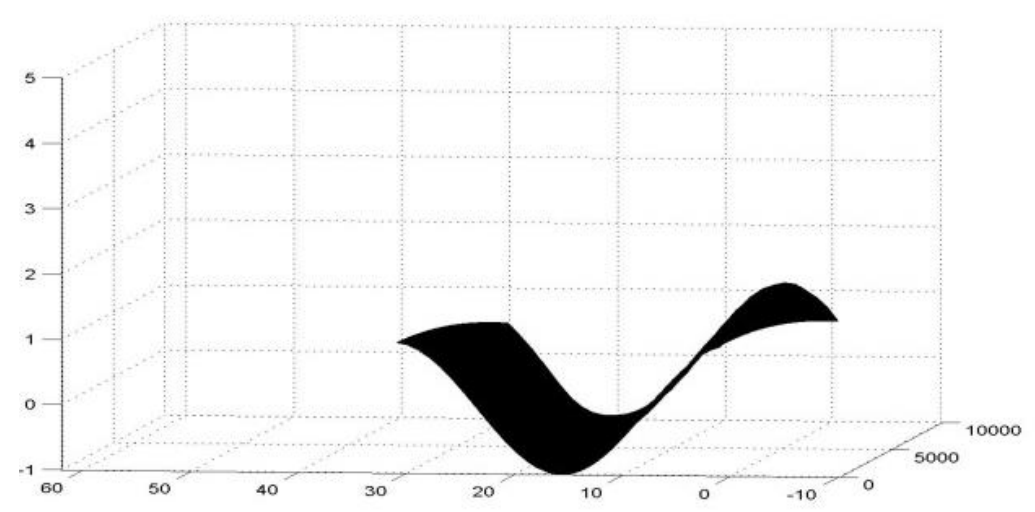

(b)

Figure 3. $t=1, \Delta t=0.0001, \Delta x=1 / 5$ graph of solution of (16) 


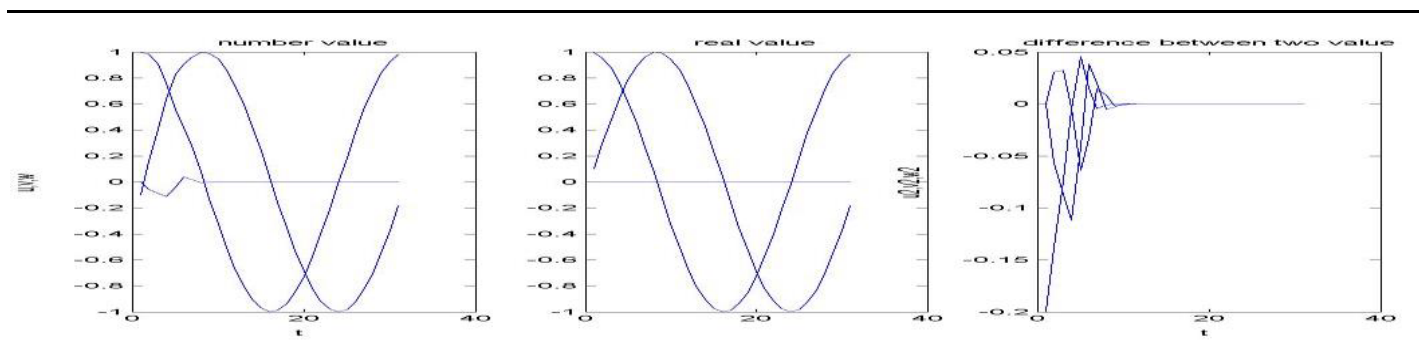

Figure 4. $\Delta t=0.1, \Delta x=1 / 5$ numerical solution and exact solution error
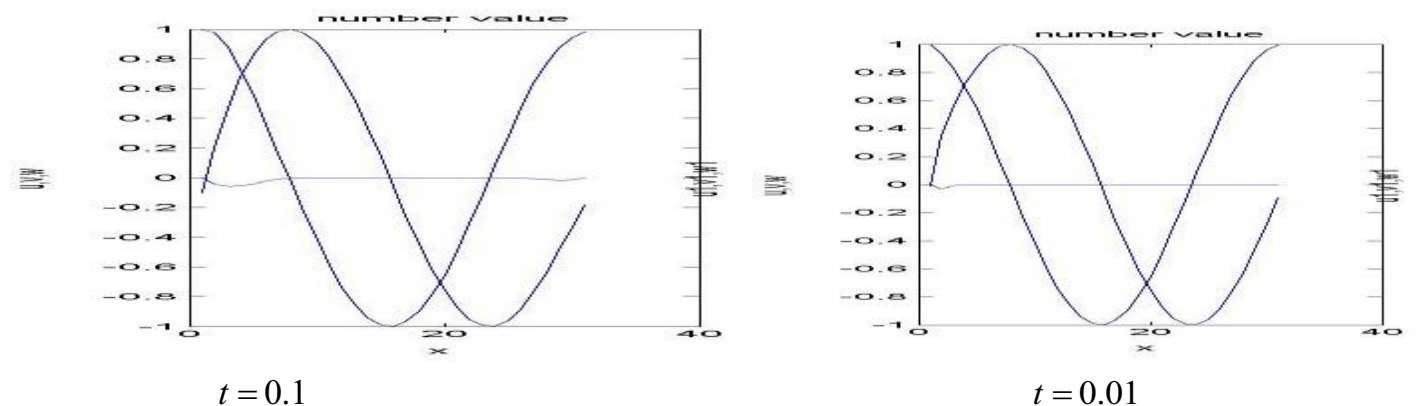

Figure 5. $\Delta t=0.0001, \Delta x=1 / 5$ graph of solution of (15) with damping

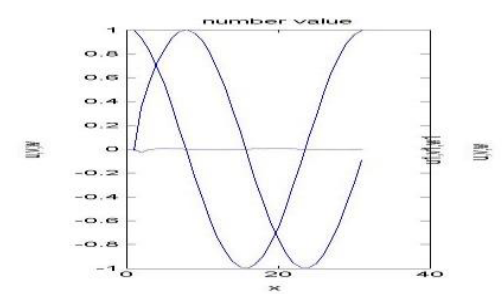

$t=0.01$



$t=0.1$

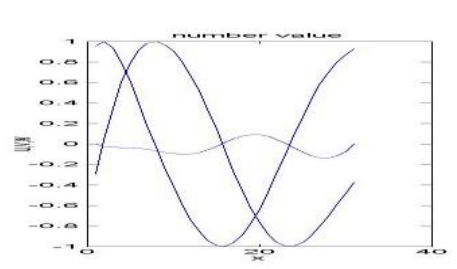

$t=0.3$

Figure 6. $\Delta t=0.0001, \Delta x=1 / 5$ graph of solution of (13) with damping

$$
\text { Where } \begin{aligned}
& u_{0}=\left(\frac{2 \sqrt{x_{1}^{2}+x_{2}^{2}}}{1+x_{1}^{2}+x_{2}^{2}}, 0, \frac{1-x_{1}^{2}-x_{2}^{2}}{1+x_{1}^{2}+x_{2}^{2}}\right), \\
& \left(x_{1}, x_{2}\right) \in[-4,4] \times[-4,4]
\end{aligned}
$$

We consider implementing (4) where the space steps $h=0.1$ and the time steps $t=0.1$ in numerical solution. Figure 1 shows the irregular change of initial value.

Observed from Figure 1 and Figure 2, for the 40 space-and-time steps, the numerical solution is a part of the round. And this conclusion may help with the later researches.

\subsection{Graph of solution of Landau-Lifshitz equation}

In 1974, Nakamura and Sasada research the following equation in the case of one-dimensional motion in [15]:

$u_{t}=u \times u_{x x}+u \times h$ where vector $h=(0,0, h), h \neq 0$
They found the following solitary wave. Tjon and Wright also found some solutions based on non-vanishing external magnetic field in 1977 [16].

$u=\left(\sqrt{1-s_{0}^{2}} \cos \xi, \sqrt{1-s_{0}^{2}} \sin \xi, s_{0}\right)$, [5].

Where $\xi=k x-w t+\varphi, s_{0}, h, w, \varphi$ are constants We consider taking $t=1$, the time-step size $\Delta t=0.0001, \Delta x=1 / 5$.Therefore, we can conclude that the method is stable.

\section{GEOMETRIC SIGNIFICANCE}

Figure 3 We can conclude that the graphs will be blowup when time $1<t<2$, thus we will improve and change designs of the numerical differential scheme. When the differential scheme is unstable, it will not be computed in the long term.

Figure 4 is the numerical solution and the exact solution error of (16) without damping when $\Delta t=0.1$, 



Figure 7. $\Delta t=0.0001, \Delta x=1 / 5$ numerical solution and exact solution graph of solution of (7)

$\Delta x=1 / 5 ;$ Figure 5 shows the graph of solution of (15) with damping.

Figure 6 is the graph of solution of (13) with damping; Figure 7 displays the numerical solution and exact solution. The graph of solution of (7) is without damping when $\Delta t=0.0001, \Delta x=1 / 5$.

\section{CONCLUSIONS}

In this paper, the author adopted a difference scheme of the Landau-Lifshitz equation by using a iteration format error about the solutions. First, the author introduced the research progress of Landau-Lifshitz equation in section 1 and established sooth solutions of the initial value problems and the difference format of Landau-Lifshitz equation with the periodic boundary condition in section 2 . Then, the author adopts numerical test and the graph of solution of Landau-Lifshitz equation in section 3 . In section 4 , the author concluded the geometric significance of Landau-Lifshitz equation with or without damping. At last, the author provided the conclusion of this research in section 5 .

\section{REFERENCES}

[1] L. D. Landau. \& E. M. Lifshitz. 1965. On the theory of the dispersion of magnetic permeability in ferromagnetic bodies. Z. Sowjetunion, 8. 1935, Reproduced in Collected Papers of L. D. Landau, Pergamon, New York, 101-114.

[2] N. Chang, J. Shatah, K. Uhlanbeck, Schrödinger maps, Comm.2000. Pure Appl. Math. 53(5): 590-602.

[3] Xiangao. Liu, 2004. Partial regularity for the Landau-Lifshitz system, Calc. Var., 20: 153-173.

[4] Changyou Wang. 2006. On Landau-Lifshitz equation in dimensions at most four. Indiana Univ. Math. J., 55(5): 1615-1644.

[5] Boling Guo \& Minchun Hong. 1993. The Landau-Lifshitz equation of the ferromagnetic spin chain and harmonic maps, Calc. Var., 1: 311-331.

[6] Boling Guo and Youde Wang, 1996. Generalized Landau-Lifshitz systems and harmonic maps. Sci. China Ser. A, 39(12): 1242-1257.

[7] Boling Guo, Yongqian Han and Ganshan Yang, 2000. Blow up problem for Landau-Lifshitz equations in two dimensions, Commun. Nonlinear Sci. Numer. Simul., 5(1): 43-44.

[8] Boling Guo, Yongqian Han and Ganshan Yang, 2001. Exact blow-up solutions for multidimensional Lan- 
dau-Lifshitz equations, Advances Mathematics in China, 30(1): 91-93.

[9] Boling Guo and Ganshan Yang, 2001. Some exact nontrivial global solutions with values in unit sphere for two-dimensional Landau-Lifshitz equations, J. Math. Physics, 42: 5223-5227.

[10] Ganshan Yang, Qianshun Chang, 2003. Limit behavior of solution for multidimensional Landau-Lifshitz equations with external magnetic field, Physics Letters A, 318(3): 270-280.

[11] Ganshan Yang and Boling Guo, 2009. Some exact solutions to multidimensional Landau-Lifshitz equation with uprush external field and anisotropy field, Nonlinear Analysis-Theory Methods Applications. 71(9): 3999-4006.

[12] Chunlai Mu, Limin Liu, Yonghong Wu, Li Yan, 2008. Some new results for the multidimensional Landau-Lifshitz equations. Phys. Lett. A, 372(43): 6469-6474.

[13] Ganshan Yang and Xiangao Liu, 2011. Spherical cone symmetric families generated by Landau-Lifshitz equation and their evolution. Sci Sin Math, 41(2): 181-196.

[14]Boling Guo, Haiyang Huang, 1999. Smooth solution of generalized system of ferromagnetic chain, Discrete and continuous dynamical systems. 5(4): 729-740.

[15]K. Nakamura and T.Sasada, 1974. Soliton and wave trains in ferromagnets. Phys. Lett. A, (48): 321-322.

[16]J. Tion and J. Wright, 1997. Soliton in the Hesenberg chain, Phys. Rev. B, (15): 3470-3476. 